\title{
HABILIDADES MOTORAS FUNDAMENTALES EN PREESCOLARES CHILENOS
}

\section{FUNDAMENTAL MOTOR SKILLS IN CHILEAN PRESCHOOLERS}

\author{
Paz Belén Fernández-Valero ${ }^{1}$ y Johana Soto-Sánchez ${ }^{1}$ \\ paz.belenfv@gmail.com; jovisoto@gmail.com \\ ${ }^{1}$ Universidad de Playa Ancha, Valparaíso, Chile
}

Envío original: 2021-07-27 Reenviado: 2021-12-03 Aceptado: 2022-01-18

Publicado: 2022-02-03

Doi: https://doi.org/10.15517/pensarmov.v20i1.47902

\begin{abstract}
RESUMEN
Las habilidades motoras fundamentales (HMF) son vinculadas como un factor clave en la promoción de estilos de vida activos y de salud a lo largo de la vida. No obstante, los resultados sobre HMF en preescolares son heterogéneos. El objetivo del estudio es describir la categorización de la puntuación obtenida en el test de HMF, así como dilucidar las diferencias por sexo y edad. Se realizó un estudio de diseño transversal, con 145 preescolares (84 varones) de 4 a 6 años (5.44 \pm 0.58 años) participantes de un programa nacional del Ministerio del Deporte. Las HMF se midieron usando la prueba de desarrollo motor grueso (segunda edición). Para el análisis por categorías de las HMF, se convirtieron en percentiles los puntajes obtenidos en la prueba presentados a través de tablas de frecuencias. Para analizar las diferencias por sexo y edad se utilizaron $U$ de Mann-Whitney y Kruskal-Wallis para aquellos sin distribución normal y los análisis de $t$ de student y ANOVA de una vía para aquellas variables con distribución normal. Los principales resultados indican que los preescolares presentan mayormente puntajes sobre el percentil ( $\geq$ p50). Los niños poseían mayores puntajes $\geq \mathrm{p} 50$ en las habilidades de locomoción, control de objetos $(p=0,007)$ y HMF total que las niñas. Niños y niñas mostraron mayores puntajes $\geq$ p50 con el aumento de la edad en habilidades de locomoción $(p=0,002)$, control de objetos $(p=0,000)$ y HMF total $(p=0,000)$. En conclusión, los preescolares chilenos presentan un adecuado desempeño en las HMF, dilucidando diferencias significativas por sexo y edad, principalmente en el control de objetos.
\end{abstract}

Palabras clave: habilidad motora, niños, habilidades, Chile. 


\begin{abstract}
Fundamental motor skills (FMS) are linked as a key factor in promoting healthy and active lifestyles throughout life. However, the results on HMF in preschool children are heterogeneous. The objective of the study is to describe the categorization of the score obtained in the FMS test, as well as to elucidate the differences by sex and age. Crosssectional design, with 145 preschoolers ( 84 boys) aged 4 to 6 years ( $5.44 \pm 0.58$ years) participating in a national program of the Ministry of Sports. FMS were measured using test of gross motor development (2nd edition). For the analysis categories of the FMS, the scores obtained in the test presented through frequency tables were converted into percentiles. To analyze the differences for sex and age we used $U$ of Mann-Whitney and Kruskal-Wallis for those without normal distribution and the analyses of t of Student's and ANOVA of one-way for those variables with normal distribution. The main results indicate that preschoolers mostly present scores above the percentile ( $\geq$ p50). Boys had higher $>$ p50 scores in the skills of locomotion, control of objects $(p=0.007)$ and total FMS than girls. Boys and girls showed higher $>$ p50 scores with increasing age in locomotion skills $(p=0.002)$, object control $(p=$ $0.000)$ and total FMS $(p=0.000)$. In conclusion, Chilean preschoolers present an adequate performance in the FMS, elucidating differences by sex and age, mainly in the control of objects.
\end{abstract}

Keywords: motor ability, children, skills, Chile.

\title{
INTRODUCCIÓN
}

La primera infancia es fundamental debido a que es un período clave para la estimulación física y mental (Kim et al., 2020) y es un momento crítico para el desarrollo de las habilidades motoras fundamentales (HMF) (Gallahue et al., 2011), siendo vinculadas como un factor clave en la promoción de estilos de vida activos y de salud a lo largo de la vida (Robinson et al., 2015; Organización Mundial de la Salud, 2019).

Las HMF son los componentes básicos necesarios para dominar movimientos complejos y específicos, permitiendo participar en deporte, juegos, danza y otras actividades deportivas, clasificadas en tres subcategorías: habilidades locomotoras (por ejemplo, correr, saltar, galopar, entre otros); control de objetos o manipulación (por ejemplo, lanzar, atrapar, patear, entre otros); y estabilidad (por ejemplo, equilibrio, balanceo, entre otros) (Goodway et al., 2019). Por tanto, la práctica y la experiencia durante la primera infancia permitirán que niños y niñas adquieren competencia de HMF (Gallahue et al., 2011). Entre los 3 y 5 años, las 
HMF deberían estar en estadios elementales de desarrollo, y al promediar 7 o 9 años, estos debieran estar siendo ejecutados de acuerdo al estadio maduro de desarrollo (Ruiz, 1994).

Durante las últimas décadas, las HMF han recibido una atención significativa debido a su asociación positiva con niveles más altos de AF (Dobell et al., 2020; Fernández-Valero et al., 2021) y de otros comportamientos y resultados relacionados con la salud (Robinson et al., 2015; Organización Mundial de la Salud, 2019), como la prevención a la obesidad (Barnett et al., 2016), disminución del comportamiento sedentario (Webster et al., 2019) y mejora la calidad del sueño (Lucena et al., 2020). Las HMF ejercen un efecto protector en la salud a fin de evitar la obesidad, excesivo tiempo de comportamiento sedentario, inadecuada calidad del sueño y condición física bajo los niveles recomendados, sin embargo, niños y niñas en todo el mundo no están logrando un adecuado desempeño de las HMF (Bolger et al., 2020), reportando una disminución en los países occidentales (Bardid et al., 2016).

Los estudios en Chile han sido escasos y han arrojado resultados diferentes. Algunas investigaciones (Poblete-Valderrama et al., 2015) han observado que los preescolares se encuentran en un nivel por debajo del esperado a su edad de sus HMF, categorizados como pobre y muy pobre evaluados con la prueba de desarrollo motor grueso- segunda edición (del inglés Test Motor Gross Development Edition-2, TGMD-2). Mientras que en otro estudio (Luna et al.,2016) encontraron que el $89 \%$ de preescolares se encuentran en un adecuado nivel de desarrollo de las HMF, evaluados con la Pauta de observación de los patrones motoras en niños de 4 a 5 años: diseño, validación, observación de desarrollo motor. Por otro lado, en otros países como Perú (Mamani-Ramos et al., 2021), México (Bermudez et al., 2018) y Sudáfrica (Tomaz et al., 2018) evaluados con la prueba de desarrollo motor grueso- segunda edición, se han observado que niños y niñas en edad preescolar presentan HMF en el promedio, por lo que probablemente las diferencias culturales pueden tener un papel influyente en la competencia de HMF entre los niños y niñas (Bolger et al., 2020). Además, el sexo y la edad también han demostrado su influencia en las HMF (Bolger et al., 2018). Los niños tienen mayores dominios de las habilidades de control de objeto y las niñas de las habilidades de locomoción (Aye et al., 2018; Bolger et al., 2018). Los niños de mayor edad tienden a tener niveles más altos en comparación con los de menor edad, en donde la puntuación total ponderada de HMF aumentan con la edad desde $37,2 \%$ entre los niños de 3 años a 76,5\% entre los de 10 años (Bolger et al., 2020).

A la luz de la importancia de las HMF y dada la falta de información descriptiva de estas habilidades en la edad preescolar, principalmente en Chile, el objetivo del presente estudio es describir la categorización de la puntuación obtenida en el test de HMF, así como dilucidar las diferencias por sexo y edad. 


\section{METODOLOGÍA}

Estudio transversal, registrado en Clinicaltrials.gov número NCT.04269135. Este estudio fue realizado entre agosto y diciembre de 2018 en la ciudad de Valparaíso y Viña del Mar de Chile, en preescolares pertenecientes al programa nacional "Crecer en Movimiento" del componente "Jugar y Aprender". Programa implementado desde el año 2014 por el Ministerio del Deporte de Chile y que consiste en clases de Educación Física estructurada, realizada por profesores de Educación Física, dos veces por semana durante 7 meses del año escolar (Kain et al., 2018).

De los 70 posibles colegios beneficiarios del componente Jugar y Aprender residentes de la región de Valparaíso, se invitó a 6 colegios a participar en el estudio. La muestra se seleccionó mediante un muestreo tipo incidental y estuvo compuesta por 145 preescolares (5.44 \pm 0.58 años) con una edad cronológica entre 4 a 6 años (84 varones y 61 niñas). La edad cronológica de los participantes fue registrada de acuerdo a la fecha de nacimiento de los niños y niñas. Además del requisito de edad, se incluyeron en el estudio preescolares sólo aquellos que manifestaron participar a través del asentimiento informado, contaban con aceptación de sus apoderados y/o tutores legales mediante el consentimiento informado. Por contraparte fueron excluidos del estudio aquellos preescolares que presentaron alguna discapacidad cognitiva, visual, auditiva y/o motora o que no pudieron seguir las instrucciones.

Este estudio contó con la aprobación del Comité de Ética e Investigación en Seres Humanos del Instituto de Nutrición y Tecnología de los Alimentos de la Universidad de Chile (INTA) (registro 9-2019), elaborado conforme con la declaración de Helsinki (1961) y a la norma legal vigente de Chile (decreto de ley 20.120).

\section{Instrumentos}

\section{Habilidades motoras fundamentales}

Las HMF se evaluaron a través de la prueba de desarrollo motor grueso- segunda edición (del inglés Test of Gross Motor Development-2, TGMD-2) (Ulrich, 2000). El test posee validez y confiabilidad para la población estadounidense, con valores de $r=0,88$ para la prueba test re-test y de 0,98 para la confiabilidad inter-evaluador. Ha sido validado en países como Brasil (Valentini, 2012) y Chile (Cano-Cappellacci et al., 2015).

Este test está diseñado para niños de 3 a 10 años y se basa originalmente en la observación de $12 \mathrm{HMF}$, comprendidas dentro de dos subescalas: 6 habilidades locomotoras (carrera, galope, salto en un pie, salto sobre un objeto, salto horizontal y desplazamiento lateral) y 6 habilidades de control de objeto (golpe de pelota estacionaria, bote de balón 
estacionaria, recepción de baloncesto, golpe de balón, lanzamiento por encima del hombro y rodar una pelota) (Ulrich, 2000).

Cada habilidad tiene de tres a cinco criterios estandarizados que al momento de evaluar se califica con un "1" si ejecuta correctamente el criterio y un "0" si no es el rendimiento esperado. El test proporciona un puntaje estándar de cada subescala y el puntaje de cada subescala es sumado para obtener un puntaje total (puntaje locomoción + control de objeto).

Los niños y niñas completaron la prueba en grupos de 8 participantes en un espacio físico destinado a la práctica deportiva de los establecimientos. Cada grupo tardó alrededor de 40 minutos. Un profesor de Educación Física proporcionó una demostración visual usando la técnica correcta, sin señalar que criterios de la habilidad eran evaluados. Los participantes fueron evaluados en vivo individualmente por un investigador capacitado y acompañados de la educadora de párvulo. No se realizó retroinformaciones sobre el rendimiento en las pruebas, solo estímulos motivacionales. Cada niño y niña realizó el test dos veces.

\section{Antropometría}

El personal de investigación capacitado en compañía de la profesora de Párvulo, midió la talla de los participantes (valor registrado en centímetros), utilizando un altímetro portátil (marca Seca, modelo 213) y el peso (valor registrado en kilogramos), utilizando una balanza (marca Seca, modelo 813). Se midió dos veces y en caso de discrepancia de más de 0,05 cm para la talla y $0,05 \mathrm{~kg}$ el peso, se midió nuevamente. Estas evaluaciones se realizaron de acuerdo a la Norma Técnica para la supervisión de niños y niñas de 0 a 9 años en la Atención Primaria de Salud (Ministerio de Salud del Gobierno de Chile, 2014).

\section{Análisis de los datos}

Los datos se analizaron utilizando el software estadístico SPSS V25.0 (Statistical Package for the Social Sciences) con un nivel de significancia establecido a priori en $p<0,05$. Antes del análisis, se exploraron los datos a fin de determinar la normalidad de estos con la prueba de Shapiro Wilk. La homogeneidad de la varianza se confirmó con la prueba de Levene. Se realizaron análisis descriptivos para edad, peso y talla.

Para la categorización de las HMF se realizó una tabla de frecuencia para todos los participantes, edad y sexo, en el cual, los puntajes brutos en cada evaluación se convirtieron en percentiles, es decir, un puntaje calculado como mayor o igual al percentil 50 ( $\geq$ p50) se consideró como habilidades con un dominio adecuado y, por el contrario, bajo el percentil 50 (<p50) se consideró como habilidades deficientes. Las HMF fueron analizadas por habilidades de locomoción, habilidades de control de objetos y HMF total. Para analizar las diferencias 
por sexo, se utilizó la prueba $t$ de Student para los datos que distribuyeron normal y $U$ de Mann-Whitney para aquellos sin distribución normal. Para analizar las diferencias por edad, se utilizó ANOVA de una vía para los datos que distribuyeron normal y Kruskal-Wallis para aquellos sin distribución normal.

\section{RESULTADOS}

En la tabla 1 se presentan las características descriptivas de los y las participantes del estudio, aquellas variables que distribuyeron normal fueron presentadas como media y desviación estándar $( \pm)$, y aquellos sin distribución normal se presentaron como mediana y percentiles (25-75). No se apreciaron diferencias por sexo en los parámetros antropométricos, sin embargo, se observan diferencias por edad.

La tabla 2 muestra la frecuencia de la categorización de HMF (habilidades de locomoción, habilidades de control de objeto y HMF total) de todos los y las participantes, por sexo y edad por separado, considerando en su construcción las HMF analizadas de acuerdo a la categoría sobre el percentil $50(\geq p 50)$ y bajo el percentil $50(<p 50)$ de los puntajes obtenidos en el TGMD-2. En primer lugar, los preescolares mostraron mayormente puntajes $\geq$ p50 en las habilidades de locomoción, control de objetos y HMF total, observando habilidades con un dominio adecuado en niños y niñas. Al comparar la categorización de HMF (habilidades de locomoción, habilidades de control de objeto y HMF total) por sexo, los niños obtuvieron mayormente puntajes $\geq$ p50, encontrando habilidades con un dominio adecuado en locomoción, control de objetos y HMF total comparado con las niñas. El análisis de comparación entre niños y niñas demostró que hay diferencias significativas en el control de objetos $(p=0,007)$.

En relación a la edad, los preescolares de 5 y 6 años obtuvieron mayormente puntajes $\geq$ p50 en las habilidades de locomoción, control de objetos y HMF total comparado con los preescolares de 4 años, quienes poseían mayormente puntajes <p50. Se encontraron diferencias significativas por edad en las habilidades de locomoción $(p=0,002)$, control de objetos $(p=0,000)$ y HMF total $(p=0,000)$. 
Tabla 1.

Descripción de las características generales de la muestra.

\begin{tabular}{|c|c|c|c|c|}
\hline & \multicolumn{4}{|c|}{ Sexo } \\
\hline & $\begin{array}{l}\text { Todos } \\
(n=145)\end{array}$ & Niños (n=84) & Niñas $(n=61)$ & $p$ \\
\hline Edad (años) ${ }^{1}$ & $5,33(5,00-5,87)$ & $5,29(4,89-5,84)$ & $5,35(5,07-5,97)$ & 0,455 \\
\hline Peso $(\mathrm{kg})^{1}$ & $\begin{array}{c}20,75(18,75- \\
23,12)\end{array}$ & $\begin{array}{c}20,52(18,43- \\
23,08)\end{array}$ & $\begin{array}{c}20,85(19,12- \\
23,12)\end{array}$ & 0,838 \\
\hline \multirow[t]{3}{*}{ Talla $(\mathrm{cm})^{2}$} & $111,46 \pm 5,87$ & $111,2 \pm 5,56$ & $111,7 \pm 6,29$ & 0,575 \\
\hline & \multicolumn{4}{|c|}{ Edad } \\
\hline & 4 años $(n=35)$ & 5 años $(n=78)$ & 6 años $(n=32)$ & $p$ \\
\hline Edad (años) ${ }^{1}$ & $4,77(4,70-4,86)$ & $5,35(5,17-5,56)$ & $6,32(6,11-6,49)$ & $0,000^{*}$ \\
\hline Peso $(k g)^{1}$ & $18,90(17,59-20,25)$ & $21,02(18,33-22,96)$ & $23,12(20,70-26,43)$ & $0,000^{*}$ \\
\hline \multirow[t]{3}{*}{ Talla $(\mathrm{cm})^{2}$} & $106,17 \pm 4,57$ & $111,51 \pm 4,72$ & $117,12 \pm 4,13$ & $0,000^{*}$ \\
\hline & \multicolumn{4}{|c|}{ Sexo } \\
\hline & $\begin{array}{c}\text { Todos } \\
(n=145)\end{array}$ & Niños (n=84) & Niñas (n=61) & $p$ \\
\hline Edad (años) ${ }^{1}$ & $5,33(5,00-5,87)$ & $5,29(4,89-5,84)$ & $5,35(5,07-5,97)$ & 0,455 \\
\hline Peso $(k g)^{1}$ & $\begin{array}{c}20,75(18,75- \\
23,12)\end{array}$ & $\begin{array}{c}20,52(18,43- \\
23,08)\end{array}$ & $\begin{array}{c}20,85(19,12- \\
23,12)\end{array}$ & 0,838 \\
\hline Talla $(\mathrm{cm})^{2}$ & $111,46 \pm 5,87$ & $111,2 \pm 5,56$ & $111,7 \pm 6,29$ & 0,575 \\
\hline
\end{tabular}

\section{Edad}

\begin{tabular}{lcccc}
\cline { 2 - 5 } & $\mathbf{4}$ años $(\mathbf{n}=\mathbf{3 5})$ & $\mathbf{5}$ años $(\mathbf{n}=\mathbf{7 8})$ & $\mathbf{6}$ años $(\mathbf{n}=\mathbf{3 2})$ & $\boldsymbol{p}$ \\
\hline Edad (años) & $4,77(4,70-4,86)$ & $5,35(5,17-5,56)$ & $6,32(6,11-6,49)$ & $0,000^{*}$
\end{tabular}

Peso $(\mathrm{kg})^{1} \quad 18,90(17,59-20,25) \quad 21,02(18,33-22,96) \quad 23,12(20,70-26,43) \quad 0,000$ *

Talla $(\mathrm{cm})^{2} \quad 106,17 \pm 4,57 \quad 111,51 \pm 4,72 \quad 117,12 \pm 4,13 \quad 0,000^{*}$

Nota: ${ }^{1}$ Mediana, (percentil 25 y 75), prueba U de Mann- Whitney (sexo) y Kruskal-Wallis (edad); ${ }^{2}$ Media \pm desviación estándar, prueba $t$ de student (sexo) y ANOVA de una vía (edad); ${ }^{*} p \leq 0.05$. Fuente: elaboración propia. 
Tabla 2.

Tabla de frecuencia de la categorización de HMF.

\begin{tabular}{|c|c|c|c|c|}
\hline & \multirow[b]{2}{*}{ Total $(n=145)$} & \multicolumn{2}{|c|}{ Sexo } & \multirow[b]{2}{*}{$p$} \\
\hline & & Niños $(n=84)$ & Niñas $(n=61)$ & \\
\hline $\begin{array}{r}\text { Locomoción } \\
<\text { p50 } \\
\geq \text { p50 }\end{array}$ & $\begin{array}{l}67(46,2 \%) \\
78(53,8 \%)\end{array}$ & $\begin{array}{l}40(27,6 \%) \\
44(30,3 \%)\end{array}$ & $\begin{array}{l}27(18,6 \%) \\
34(23,4 \%)\end{array}$ & 0,651 \\
\hline $\begin{array}{c}\text { Control de Objetos } \\
\quad<\text { p50 } \\
\geq \text { p50 }\end{array}$ & $\begin{array}{l}64(44,1 \%) \\
81(55,9 \%)\end{array}$ & $\begin{array}{l}31(21,4 \%) \\
53(36,6 \%)\end{array}$ & $\begin{array}{l}33(22,8 \%) \\
28(19,3 \%)\end{array}$ & $0,007^{*}$ \\
\hline $\begin{array}{r}\text { HMF total } \\
<\mathrm{p} 50 \\
\geq \mathrm{p} 50 \\
\end{array}$ & $\begin{array}{l}70(48,3 \%) \\
75(51,7 \%) \\
\end{array}$ & $\begin{array}{l}36(24,8 \%) \\
48(33,1 \%)\end{array}$ & $\begin{array}{l}34(23,4 \%) \\
27(18,6 \%) \\
\end{array}$ & 0,141 \\
\hline & & Edad & & \\
\hline & 4 años (n=35) & 5 años $(n=78)$ & 6 años $(n=32)$ & $p$ \\
\hline $\begin{array}{r}\text { Locomoción } \\
<\text { p50 } \\
\geq \text { p50 }\end{array}$ & $\begin{array}{c}23(15,9 \%) \\
12(8,3 \%)\end{array}$ & $\begin{array}{l}33(22,8 \%) \\
45(31,0 \%)\end{array}$ & $\begin{array}{c}11(7,6 \%) \\
21(14,5 \%)\end{array}$ & $0,002^{*}$ \\
\hline $\begin{array}{c}\text { Control de Objetos } \\
\quad<p 50 \\
\quad \geq \text { p50 }\end{array}$ & $\begin{array}{c}26(17,9 \%) \\
9(6,2 \%)\end{array}$ & $\begin{array}{l}32(22,1 \%) \\
46(31,7 \%)\end{array}$ & $\begin{array}{c}6(4,1 \%) \\
26(17,9 \%)\end{array}$ & $0,000^{*}$ \\
\hline $\begin{array}{r}\text { HMF total } \\
<p 50 \\
\geq p 50\end{array}$ & $\begin{array}{c}28(19,3 \%) \\
7(4,8 \%)\end{array}$ & $\begin{array}{l}35(24,1 \%) \\
43(29,7 \%)\end{array}$ & $\begin{array}{c}7(4,8 \%) \\
25(51,7 \%)\end{array}$ & $0,000^{*}$ \\
\hline
\end{tabular}

Nota: Prueba U de Mann- Whitney (sexo) y Kruskal-Wallis (edad); número de participantes (porcentaje); <p50: bajo el percentil 50; $\geq$ p50: sobre el percentil 50; HMF total: habilidades motoras fundamentales total; * $p \leq 0.05$. Fuente: Elaboración propia.

\section{DISCUSIÓN}

Este estudio tuvo como objetivo describir la categorización de la puntuación obtenida en el test de HMF, así como dilucidar las diferencias por sexo y edad. Los resultados de este estudio mostraron que los preescolares poseen mayormente puntajes $\geq p 50$, observando diferencias significativas por sexo en el control de objetos y por edad en locomoción, control de objetos y HMF total.

Los hallazgos de este estudio son similares a otros estudios realizados en Perú y Sudáfrica (Mamani-Ramos et al., 2021; Tomaz et al., 2018) y principalmente a un estudio realizado en Chile, en la ciudad de Concepción (592 kilómetros de Valparaíso) (Luna et al., 2016), observando que los preescolares evaluados presentaron puntajes que les permiten ubicarse en los rangos esperados para su edad cronológica. Sin embargo, se han encontrado resultados que informan niveles bajos de HMF entre los niños y niñas (Bolger et al., 2018; 
Poblete-Valderrama et al., 2015; O`Brien et al., 2016). Es importante señalar que los niños y niñas de este estudio eran beneficiarios del componente "Jugar y Aprender" del programa "Crecer en movimiento" del Instituto Nacional de Deporte, por tanto, tenían alrededor de 180 minutos de actividad física programada a la semana con un profesional idóneo del área. Esto podría explicar el rendimiento en las HMF, ya que se han demostrado mejoras cuando los niños y niñas son instruidos directamente por personas especialistas en HMF (por ejemplo, profesores de Educación Física) (Engel et al., 2018).

En este estudio, los niños presentaban mayormente puntajes en el $\geq$ p50 en las habilidades de locomoción, control de objetos y HMF total en comparación con las niñas, lo que está en línea con estudios anteriores (Aye et al., 2018; Tomaz et al., 2018). Principalmente, se encontraron diferencias significativas para las habilidades de control de objetos concordando con los resultados de diversos estudios (Bolger et al., 2020; Mohammadi et al., 2017) que han utilizado diferentes instrumentos para evaluar las HMF (por ejemplo, Movement Assessment Battery For Children-Second Edition; TGMD-3). Esto demuestra que incluso cuando se usan diferentes instrumentos, los niños tienen puntajes más altos en control de objetos. Por otro lado, la diferencia entre sexo se puede deber primordialmente a la influencia de las expectativas del rol de la familia, el medio ambiente (Zeng et al., 2019), y el tipo de actividades en las que participan los niños y niñas (Bolger et al., 2020), ya que desde un punto de vista biológico, pueden observarse diferencias de sexo en altura y peso, pero son mínimas. Los físicos de los niños y niñas en edad preescolar son notablemente similares (Goodway et al., 2019). Por lo tanto, esta diferencia esta influenciada en gran medida por los factores sociales y ambientales como los padres, ya que tienen una gran influencia en los deportes en los que participan sus hijos, incentivándolos a participar en juegos y deportes que requieran balón, sin embargo, mucho de los padres consideran que los juegos y/o actividades que implican objetos o balón son demasiado difíciles para las niñas, y por lo tanto, no incentivan a sus hijas a participar en ellas (Yang et al., 2015). No obstante, se ha observado que las niñas realizan significativamente mejores habilidades de locomoción que los niños (Aye et al., 2018; Bolger et al., 2018) lo que difiere con nuestro estudio.

La comparación entre grupos de edad mostró que hay diferencias significativas en las habilidades de locomoción, control de objetos y HMF total, en donde, los preescolares de mayor edad poseían mayormente puntajes $\geq$ p50 en comparación con los preescolares de 4 años. Estos resultados son consistentes con los reportados por Mohammadi et al. (2017); Bolger et al. (2018); Fernández-Valero et al. (2021), que demostraron que las HMF tiende a mejorar con el aumento de la edad. Esto se puede deber principalmente al estadio en que se encuentran las HMF a su edad, debido que entre los 3 y 5 años, las HMF deberían estar en 
estadios elementales de desarrollo, y al promediar 7 u 9 años, estos debieran estar siendo ejecutados de acuerdo al estadio maduro de desarrollo (Ruiz, 1994). Por otro lado, hay que considerar que a medida que los niños y niñas se desarrollan, hay un mayor crecimiento físico y una mayor fuerza en relación con la masa corporal (Cohen et al., 2015). Además, hay factores externos como la práctica, el estímulo y la instrucción que pueden diferir entre el desempeño de los niños cuando crecen (Mohammadi et al., 2017). Sin embargo, Yang et al. (2015) no encontró diferencias entre los grupos de edad de 5 y 6 años.

Los años preescolares son vitales para estimular las HMF (Goodway et al., 2019) y equiparar la brecha entre niños y niñas, lo que hace que estos hallazgos tengan implicaciones importantes para las educadoras de párvulos y profesores de Educación Física que están involucrados en los programas de cuidado y desarrollo de la primera infancia. En primer lugar, es esencial que un profesional especialista en el área del desarrollo motor enfatice en una variedad de oportunidades de practica que implique actividades que permitan a los niños y niñas moverse por el espacio o mover su cuerpo de un punto a otro como correr, saltar a pies juntos, caminar, saltar en un pie y galopear, y actividades que impliquen manipular o controlar objetos como lanzar, patear, atrapar, golpear, rodar y patear/driblear con diversos materiales, progresando de lo simple a lo complejo. Los intereses y las habilidades de los niños y niñas son similares, sin necesidad de actividades separadas durante este período, por lo que es necesario involucrar a las niñas en actividades enfocadas en habilidades de locomoción y control de objetos de igual forma que los niños, sin hacer diferencias. Por otro lado, al observar en nuestro estudio que niños y niñas de edad preescolar tienen un dominio adecuado de las HMF, nuestros resultados demuestran la relevancia que tiene para un niño y niña contar con un profesional especialista en el ámbito del desarrollo motor, ya que el logro de la etapa competente en estas habilidades está muy influenciado por las oportunidades de práctica, estímulo e instrucción que pueda proporcionar un profesional idóneo en el área en un entorno que fomenta el aprendizaje (Goodway et al., 2019). No obstante, esta no es la realidad de todos los niños y niñas en edad preescolar que asisten a jardines de cuidado infantil. Lo anterior resulta crucial porque los niveles de HMF pueden contribuir a aumentar los niveles de $A F$, en el cual, los bajos niveles de AF parece ser el mayor determinante de la obesidad infantil (Macedo et al., 2019).

La fortaleza de este estudio fue que las HMF fueron evaluadas con un instrumento estandarizado y validado orientado en el proceso. La principal limitación de este estudio fue que la muestra era solo de la región de Valparaíso, Chile. Se necesitan más estudios que involucren tamaños de muestra más grande de todo el país. Además, investigaciones futuras podrían agregar un grupo control para comparar niños y niñas que no pertenezcan al 
programa crecer en movimiento y encuestas a los padres sobre qué actividades realizan sus hijos e hijas a diario.

\section{CONCLUSIÓN}

Los preescolares chilenos ( 4 a 6 años) presentaron mayormente puntajes $\geq$ p50 de las HMF, es decir, presentan habilidades con un dominio adecuado. Se encontraron diferencias significativas por sexo y edad, siendo los niños y los preescolares con 6 años los que presentan un mayor dominio de las habilidades de control de objetos. Estos hallazgos proporcionan información valiosa que se puede utilizar para establecer referencias normativas para las HMF de los preescolares chilenos en estudios futuros. Con estos resultados se sugiere considerar en las clases de Educación Física, al momento de estimular las HMF, las diferencias por sexo para involucrar a las niñas a una mayor participación de actividades enfocadas en habilidades de locomoción y control de objetos. Es ideal que en la edad preescolar cuenten con un profesional especialista en el desarrollo motor a fin de permitir que niños y niñas alcancen sus niveles adecuados de HMF.

\section{FINANCIAMIENTO}

Este estudio se enmarca en el proyecto financiado por la Comisión Nacional de Investigación Científica y Tecnológica CONICYT denominado "Preschool Fit-healthy and Smart PREFIT-Chile Study Linking Physical Fitness to non invasive health-related markers and Executive Function" (REDI170474). Por la Dirección General de Investigación de la Universidad de Playa Ancha (EFI01 2018-2019). Adicionalmente, este estudio fue financiado en el marco del Concurso de Financiamiento de Tesis de Postgrado de la Dirección General de Investigación de la Universidad de Playa Ancha y del Proyecto Nº EFI03 2017-2018.

\section{AGRADECIMIENTO}

Los autores agradecen el apoyo de los niños, padres, profesores y cuerpo directivo de los establecimientos Ana Maria Janer, Mar Abierto, Padre Zanandrea, República Paraguay, República de México y Canal Beagle. Igualmente, se agradece al programa "Crecer en Movimiento", específicamente al componente "Jugar y Aprender" del Instituto Nacional del Deporte del Ministerio del Ministerio del Deporte.

\section{CONFLICTO DE INTERESES}

Los autores declaran no tener ningún conflicto de intereses. 


\section{REFERENCIAS}

Aye, T., Kuramoto-Ahuja, T., Sato, T., Sadakiyo, K., Watanabe, M., y Maruyama, H. (2018). Gross motor skill development of kindergarten children in Japan. The Journal of Physical Therapy Science, 30(5), 711-715. https://doi.org/10.1589/jpts.30.711

Bardid, F., Lenoir, M., Huyben, F., De Martelaer, K., Seghers, J., Goodway, J., y Deconinck, F. (2016). The effectiveness of a community-based fundamental motor skill intervention in children aged 3-8 years: Results of the "Multimove for Kids" project. Journal of Science and Mededicine in Sport, 20(2), 184-189. https://doi.org/10.1016/j.jsams.2016.07.005

Barnett, L., Lai, S., Veldman, S., Hardy, L., Cliff, D., Morgan, P., ... Okely, A. (2016). Correlates of Gross Motor Competence in Children and Adolescents: A Systematic Review and Meta-Analysis. Sports Med, 46(11), 1663-1688. https://doi.org/10.1007/s40279-016$\underline{0495-z}$

Bermudez, M., Poblete-Valderrama, F., Pineda-Espejel, A., Castro, N., y Inostroza, F. (2018). Nivel de desarrollo motor grueso en preescolares de México sin profesores de educación física. Revista Ciencias de la Actividad Física, 19(1), 75-81. https://doi.org/10.29035/rcaf.19.1.8

Bolger, L., Bolger, L., O`Neill, C., Coughlan, E., O’Brien, W., Lacey, S., y Burns, C. (2018). Age and sex differences in fundamental movement skills among a cohort of Irish school children. Journal of Motor Learning and Development, 6(1), 81-100. https://doi.org/10.1123/jmld.2017-0003

Bolger, L., Bolger, L., O`Neill, C., Coughlan, E., O’Brien, W., Lacey, S., ... Bardid, F. (2020). Global levels of fundamental motor skills in children: A systematic review. Journal of Sports Sciences, 39(7), 717-753. https://doi.org/10.1080/02640414.2020.1841405

Cano-Cappellacci, M., Leyton, F., y Duran, J. (2015). Content validity and reliability of test of gross motor development in Chilean children. Rev Saúde Pública, 49. https://doi.org/10.1590/S0034-8910.2015049005724

Cohen, K., Morgan, P., Plotnikoff, R., Barnett, L., y Lubans, D. (2015). Improvements in fundamental movement skill competency mediate the effect of the SCORES intervention on physical activity and cardiorespiratory fitness in children. Journal of Sports Sciences, 33(18), 1908-1918. https://doi.org/10.1080/02640414.2015.1017734

Dobell, A., Pringle, A., Faghy, M. y Roscoe, C. (2020). Fundamental movement skills and accelerometer-measured physical activity levels during early childhood: A systematic review. Children, 7(11), 224. https://doi.org/10.3390/children7110224

Engel, A., Broderick, C., van Doorn, N., Hardy, L., y Parmenter, B. (2018). Exploring the Relationship Between Fundamental Motor Skill Interventions and Physical Activity 
Levels in Children: A Systematic Review and Meta-analysis. Sports Med, 48(8), 18451857. https://doi.org/10.1007/s40279-018-0923-3

Fernández- Valero, P., Soto-Sánchez, J., Páez, J., Leyton- Dinamarca, B., Kain, J., Hurtado, J., y Reyes-Amigo, T. (2021). Fundamental movement skills and physical activity recommendations for preschool children. Journal of Physical Education and Sport, 21(6), 3286-3293. https://doi.org/10.7752/jpes.2021.s6436

Gallahue, D., Ozmun, J., y Goodway, J. (2011). Understanding motor development: Infants, children, adolescents, adults. McGraw-Hill.

Goodway, J., Ozmun, J., y Gallahue, D. (2019). Understanding motor development. Infants, children, adolescents, adults (8 ed.). Jones \& Bartlett Learning

Kain, J., Leyton, B., Soto-Sanchez, J., y Concha, F. (2018). In preschool children, physical activity during school time can significantly increase by intensifying locomotor activities during physical education classes. BMC Res Notes, 11(1). https://doi.org/10.1186/s13104-018-3536-x

Kim, H., Ma, J., Harada, K., Lee, S. y Gu, Y. (2020). Associations between adhrence to combinations of 24-h movement guidelines and overweight and obesity in Japanese preschool children. International Journal of Environmental Research and Public Health, 17(24). https://doi.org/10.3390/ijerph17249320

Lucena, C., Clark, C., Ribeiro, P., Mota, J., y Duncan, M. (2020). Association between Compliance with the 24-Hour Movement Guidelines and Fundamental Movement Skills in Preschoolers: A Network Perspective. International Journal of Environmental Research and Public Health, 17(15). https://doi.org/10.3390/ijerph17155443

Luna, P., Rodríguez, V., Sandoval, M., y Carreño, M. (2016). Análisis de patrones motores fundamentales en niños de 4 y 5 años de colegios particulares subvencionados de Concepción. Revista Ciencias de la Actividad Física, 17(2), 19-28. http://revistacaf.ucm.cl/article/view/96/101

Macedo, F., Pinheiro, R., Cintra, J., Nogueira, W., Marques, A., Deana, N. y Alves, N. (2019). Influencia de la práctica de la actividad física y del comportamiento dietético en el índice de masa corporal de niños y adolescentes: Revisión de literatura. Revista Chilena de Nutrición, 46(3), 343-351. http://dx.doi.org/10.4067/S0717-75182019000300343

Mamani-Ramos, A., Dextre-Mendoza, C., Lava, J., Ticona, G., Quispe, L., Torres-Cruz, F., ... Fuentes-López, J. (2021). Gross motor development in preschoolers on the islands of Lake Titicaca (3810 m.s.n.m.), Puno, Peru. Retos, 39, 592-597. https://doi.org/10.47197/retos.v0i39.79522 
Ministerio de Salud del Gobierno de Chile. (2014). Norma técnica para la supervisión de niños y niñas de 0 a 9 años en la Atención Primaria de Salud. https://www.minsal.cl/sites/default/files/files/2014 Norma\%20Técnica\%20para\%20la\% 20supervisión $\% 20$ de $\% 20$ niños $\% 20 y \% 20$ niñas $\% 20$ de $\% 200 \% 20 a \% 209 \% 20$ en $\% 20$ APS web(1).pdf

Mohammadi, F., Bahram, A., Khalaji, H. y Ghadiri, F. (2017). Determining motor development status of 3-10 year-old children in Ahvaz city using TGMD-3 test. International Journal of Basis Science in Medicine, 2(3), 139-146. https://doi.org/10.15171/ijbsm.2017.26

O'Brien, W., Belton, S., y Issartel, J. (2016). Fundamental movement skill proficiency amongst adolescent youth. Physical Education and Sport Pedagogy, 21(6), 557-571. https://doi.org/10.1080/17408989.2015.1017451

Organización Mundial de la Salud (2019). Directrices sobre la actividad física, el comportamiento sedentario $y$ el sueño para menores de 5 años. https://iris.paho.org/bitstream/handle/10665.2/51805/9789275321836 spa.pdf? sequen ce5\&isAllowedy

Poblete-Valderrama, F., Morilla, C., y Quintana, C. (2015). Nivel de desarrollo motor grueso en pre-escolares sin intervención de profesores de Educación Física, Valdivia. Revista Horizonte Ciencias de la Actividad Física, 6(2), 33-40. http://revistahorizonte.ulagos.cl/index.php/horizonte/article/view/81

Robinson, L., Stodden, D., Barnett, L., Lopes, V., Logan, S., Rodrigues, L., y D'Hondt, E. (2015). Motor Competence and its Effect on Positive Developmental Trajectories of Health. Sports Med, 45(9), 1273-1284. https://doi.org/10.1007/s40279-015-0351-6

Ruiz, L. (1994). Desarrollo motor y actividades físicas (3 ed.). Madrid, España: GYMNOS.

Tomaz, S., Jones, R., Hinkley, T., Bernstein, S., Twine, R., Kahn, K., ... Draper, C. (2018). Gross motor skils of South African preschool-aged children across different income settings. Journal of Science and Medicine in Sport, 22(6), 689-694. https://doi.org/10.1016/j.jsams.2018.12.009

Ulrich, D. (2000). Test of gross motor development. Examiner's manual (2 ed.). https://33202576.weebly.com/uploads/1/4/6/8/14680198/tgmd-2-2.pdf

Valentini, N. (2012). Validity and reliability of the TGMD-2 for Brazilian children. Journal of Motor Behavior, 44(4), 275-280. https://doi.org/10.1080/00222895.2012.700967

Webster, E., Martin, C., y Staiano, A. (2019). Fundamental motor skills, screen-time, and physical activity in preschoolers. Journal of Sport Health Science, 8(2), 114-121. https://doi.org/10.1016/j.jshs.2018.11.006 
Yang, S., Lin, S. y Tsai, C. (2015). Effect of sex, age, and BMI on the development of locomotor skills and object control skills among preschool children. Perceptual \& Motor Skills, 121(3), 873-888. https://doi.org/10.2466/10.PMS.121c29x0

Zeng, N., Johnson, S., Boles, R. y Bellows, L. (2019). Social-ecological correlates of fundamental movement skills in young children. Journal of Sport and Health Science, 8(2), 122-129. https://doi.org/10.1016/j.jshs.2019.01.001 